\title{
Dielectric and Spectroscopic Investigations of Amorphous Captopril
}

\author{
Sailaja Urpayil ${ }^{1,2}$, M.Shahin Thayyil. ${ }^{1}$ \\ ${ }^{1}$ Department of Physics, University of Calicut, Kerala, INDIA \\ 2 Department of Physics MES Keveeyam College, Valancherry, Kerala, INDIA
}

\begin{abstract}
Captopril, an orally active dipeptide analogue was introduced in 1977 and used for the treatment of hypertension and in patients having ischaemic heart disease. Since the bioavailability of the drug is low, an alternative method to increase its bioavailability and solubility is by changing the drug in to amorphous form. We used Differential Scanning Calorimetry (DSC) for studying the glass forming ability of the drug. The sample was found to be a very good glass former. Based on the DSC analysis we have used broadband dielectric spectroscopy (BDS) for studying the drug in the super cooled and glassy state. BDS is an effective tool to probe the molecular dynamics in the super cooled and glassy state. Molecular mobility is found to be present even in the glassy state of this active pharmaceutical ingredient (API) which is responsible for the instability. The sample is highly unstable in the amorphous state and our aim is to study the factors responsible for instability of this API. Our FTIR study showed that hydrogen bonding is stronger in the amorphous state than that of crystalline state of this API.
\end{abstract}

Abbreviations. API Active pharmaceutical ingredient; BDS Broadband dielectric spectroscopy; ACE angiotensinconverting enzyme; DSC Differential scanning calorimetry; FTIR fourier transform infrared spectroscopy; VFTH VogelFulcher-Tamman-Hesse. XRPD X-Ray Powder diffraction.

Keywords. Amorphous state, Bioavailability, Broadband dielectric spectroscopy, Differential scanning calorimetry, Molecular dynamics

\section{INTRODUCTION}

Glass is familiar to mankind for years. But the theory behind glass formation is a crucial one to scientific community. Complete theory about glass formation is still unknown. Glass was considered as a transparent product which is formed due to the fusion of minerals. The important concepts involved in the glass formation were the composition of the glass material, method of formation and the metastability of the state with respect to the stable crystalline state. In olden days it was believed that glass was formed from inorganic substances. For scientific purposes many organic substances including synthetic polymers can be converted into glass. The idea that glass as a super cooled liquid was first given by Tamman [1]. In glassy state different relaxation phenomena can be observed due to molecular motions present. Occurrence of the relaxation in the super cooled region is due to the increase in viscosity when the temperature decreases. Out of the various relaxations phenomenon the slowest one is the primary relaxation or $\alpha$-relaxation which is due to the cooperative rearrangement of molecules above the glass transition temperature [2]. Below the glass transition temperature, various other relaxations often called the secondary relaxations are observed.

In 1971 Johari and Goldstein found that secondary relaxations are considered as the characteristic property of the liquids, and it is due to the motion of the entire molecule. This relaxation is known as J-G relaxation or $\beta$-relaxation and it is found to be universal [3]. Since glass transition is a kinetic phenomena on cooling a liquid, the relaxation times increases in a non Arrhenius manner. Another common feature of glass transition is a step like change in the specific heat. The magnitude of the step like change is connected to the departure from Arrhenius behavior, called the fragility. There are two types of glasses, "strong" and "fragile". The strong systems show strong resistance against structural degradation and shows Arrhenius behavior, and fragile glassformers are having nondirectional interatomic or intermolecular bonds and shows deviation from Arrhenius behaviour [4]. Glass transition can be applied to determine the physical and chemical properties of many active pharmaceutical ingredients (APIs). The amorphous solids are very important in the pharmaceutical point of view because they have higher solubility, higher dissolution rate, and better compression characteristics than the corresponding crystalline counterpart. The physical instability of the amorphous form due to higher molecular mobility and free energy caused the system to revert to the more stable crystalline form which will result poor product performance [5-7]. Disordered amorphous material will dissolve faster and has a greater solubility and bioavailability than the corresponding ordered crystalline counterpart [8], [9]. Amorphous form of a drug often shows an improved therapeutic activity [10]. Preparation of the drug in the amorphous form can avoid the use of excipients, which is usually used to shape drugs in to tablets [11]. Even, in the case of highly soluble crystalline API, amorphous form is a good alternative. Recently Allie et al showed that crystallization can be determined by intramolecular motion involving the $\beta_{\mathrm{a}^{-}}$relaxation mode and not by the molecular motion responsible for the $\alpha_{\mathrm{a}}$ - relaxation mode [12]. So it is important to study the molecular dynamics in the glassy state [13], [14]. Molecular mobility in the amorphous state is very important to predict the physical and chemical stability of the API [5]. The secondary relaxation present in the glassy state is very important to predict the stability of API [15]. Differential scanning calorimetry (DSC) can be used to study the thermal properties of several drugs in their crystalline and amorphous form [8]. Broadband dielectric spectroscopy is an effective tool to probe the molecular dynamics in the super cooled and glassy state of API and was recently employed in pharmaceutical research [16]. 
Captopril is selected for the current study as a model drug. Captopril an orally active dipeptide analogue was introduced in 1977 and quickly gained wide usage. The pharmacology of Captoril is described as prototype, since most of its effects are class effects common to all angiotensin-converting enzyme (ACE) inhibitor. It lowers blood pressure. This can be safely used in patients with ischaemic heart diseases. Pharmacokinetics of Captopril is about 70\% of orally administered Captopril is absorbed. Presence of food in the stomach reduces its bioavailability. Penetration in to brain is poor. It is partially excreted unchanged in the urine. The plasma $t_{1 / 2}$ is $\sim 2$ hours, but action lasts for $6-12$ hours [17]. So it is essential to increase the bioavailability of this novel drug.

Our DSC study shows that Captopril is a non crystallizing compound. Based on the result of DSC study, we explored the molecular dynamics of super cooled and the glassy state of this API with the help of BDS. From dielectric studies onset crystallization was observed at $343.15 \mathrm{~K}$. Besides secondary processes the structural relaxation can also be responsible for the devitrification of this drug [18]. Molecular mobility is found to be present below glass transition temperature $T_{\mathrm{g}}$. Fragility of this API is found to be 84 proving it is a fragile glass former often considered physically unstable [4]. Spectroscopic study was used to investigate the hydrogen-bonding interaction in the crystalline and amorphous state [1921], [16]. In Captopril the shifting of the carbonyl peak towards the lower frequency in the amorphous state shows stronger hydrogen bonding in the amorphous phase.

\section{MATERIALS AND METHODS}

\subsection{Materials}

Captopril,CAS NO 6271-86-2 was purchased from Sigma Aldrich (purity $\geq 98 \%$ ) and molecular mass of $M_{W}=217.29$

g. mol $^{-1}$.Captopril is (2S)-1-[(2s)-2-methyl- 3-sulfanyl propanoyl] pyrrolidine-2-carboxylic acid. Its empirical formula is $\mathrm{C}_{9} \mathrm{H}_{15} \mathrm{NO}_{3} \mathrm{~S}$. The chemical structure is presented in fig.1.The purchased material was used without further purification.

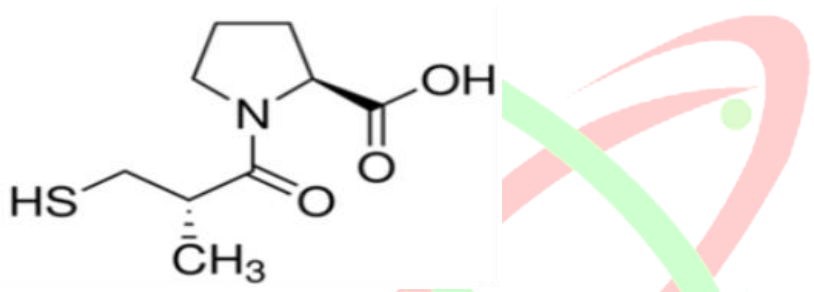

Fig. 1. The chemical structure of Captopril.

\subsection{Assay}

Assay of Captopril is done by titration method and the value is obtained as 99.26 percent of $\mathrm{C}_{9} \mathrm{H}_{15} \mathrm{NO}_{3} \mathrm{~S}$. And it is found to be within the limits as specified in IP [22].

\subsection{Differential Scanning Calorimetry (DSC)}

Calorimetric response of the sample was measured using a DSC instrument $\left(821^{\mathrm{e}}\right.$ Metler-Toledo GmbH) operating with STAR $^{\mathrm{e}}$ software version 9.1 and equipped with an intra cooler. The instrument was calibrated by using indium. The samples $(3-5 \mathrm{mg})$ were analyzed under dry nitrogen purge $(50 \mathrm{ml} / \mathrm{min})$ in a sealed and pinhole aluminum pan. The sample is heated from room temperature to $122^{\circ} \mathrm{C}$ and held for 5 minutes, then the sample is cooled to $\left(-50^{\circ} \mathrm{C}\right)$ and held for 15 minutes, then the sample is again heated to $127^{\circ} \mathrm{C}$ a constant heating and cooling rate of $10^{\circ} \mathrm{C} / \mathrm{min}$ is used.

\subsection{X-Ray Powder diffraction (XRPD)}

X-ray Powder diffraction measurements were used to confirm the crystalline nature of the sample. A XPERT-PRO diffractometer system with a rotating anode $\mathrm{Cu} \mathrm{K \alpha}$ was used and scans were taken between $5^{0}$ to $100^{\circ}{ }_{2} \theta$.

\subsection{Fourier Transform Infrared (FTIR) Spectroscopy}

FTIR spectra were collected on a FTIR microscope (Perkin Elmer, Model: Synthesis Monitoring System) for amorphous system and FTIR (Nicolet instruments corporation USA Model MAGNA 550) for crystalline state.

\subsection{Broadband Dielectric Spectroscopy (BDS) measurements}

Dielectric measurements of Captopril at ambient pressure were carried out using Novo-Control GMBH alpha analyzer over a wide frequency range of $10^{-2}$ to $10^{7} \mathrm{~Hz}$. The temperature was controlled using nitrogen gas cryostat with temperature stability better than $0.1 \mathrm{~K}$. The sample was placed between two stainless steel electrodes of the capacitor with a gap of $0.20 \mathrm{~mm}$ and 
diameter $30 \mathrm{~mm}$. Teflon is used as the spacer. Dielectric measurements of the sample were performed after its vitrification by fast cooling $(10 \mathrm{~K} / \mathrm{min})$ from few degrees above the melting point $\left(\mathrm{T}_{\mathrm{m}}=380 \mathrm{~K}\right)$. The dielectric spectra of Captopril were collected in a wide temperature range from $123.15 \mathrm{~K}$ to $373.15 \mathrm{~K}$ in different steps: 123.15 to $263.15 \mathrm{~K}$ in steps of $10 \mathrm{~K}$, whereas in temperature range from $263.15 \mathrm{~K}$ to $305.15 \mathrm{~K}$ in steps of $2 \mathrm{~K}, 305.15$ to $333.15 \mathrm{~K}$ in steps of $5 \mathrm{~K}$ and from 343.15 to373.15K in steps of $10 \mathrm{~K}$.

\section{RESULTS AND DISCUSSION}

\subsection{Thermal analysis}

From DSC study $\mathrm{T}_{\mathrm{g}}$ has been reported as the onset value of the glass transition event. It was observed that the glass transition temperature of the amorphous sample was $273.91 \mathrm{~K}$. This is in close agreement with the value obtained for the DSC measurement of Pajula et al [23]. The sample did not crystallize at all during the cooling and heating treatment. Hence it is classified as a non- crystallizing compound. $\mathrm{T}_{\mathrm{m}}$ is the melting temperature and measured as $380.34 \mathrm{~K}$.

\subsection{Spectral Variations of Captopril in crystalline and amorphous systems.}

IR spectroscopy is used for investigating the nature of hydrogen bonding in crystalline and amorphous Captopril. Hydrogen bonding can occur in any system containing a proton donor group $(\mathrm{X}-\mathrm{H})$ and a proton acceptor(Y) if the $s$ orbital of the proton can effectively overlap the por pi orbital of the acceptor group. The common proton donor groups in organic molecules are carbonyl, hydroxyl, amine, or amide groups. Common proton acceptor atoms are oxygen, nitrogen, and the halogens. Hydrogen bonding alters the force constants of both groups. When bonding occur the X-H stretching band moves to lower frequencies (longer wavelengths). The FTIR analysis of crystalline Captopril showed a $\mathrm{C}=\mathrm{O}$ vibration at $1748 \mathrm{~cm}^{-1}$. In Amorphous $\mathrm{C}=\mathrm{O}$ band shifts down to lower wave number $1733 \mathrm{~cm}^{-1}$. The hydrogen bond formation reduces the carbonyl peak position [20], [24]. So in the case of Captopril hydrogen bonding is stronger in the amorphous state than that of the crystalline state.

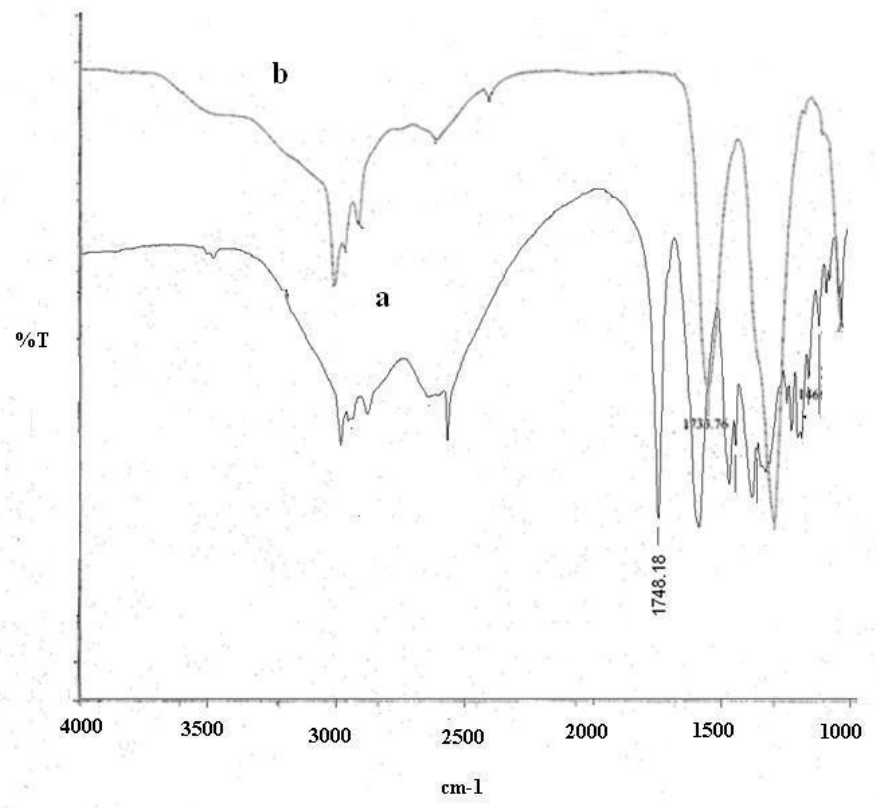

Fig. 2. FTIR spectra in $\mathrm{C}=\mathrm{O}$ stretching vibration region (a) crystalline Captopril (b) amorphous Captopril

\subsection{XRPD Result}

The sharp peaks in fig. 3 show the crystalline nature Captopril. 
IOSR Journal of Pharmacy

Vol. 2, Issue 3, May-June, 2012, pp.479-484

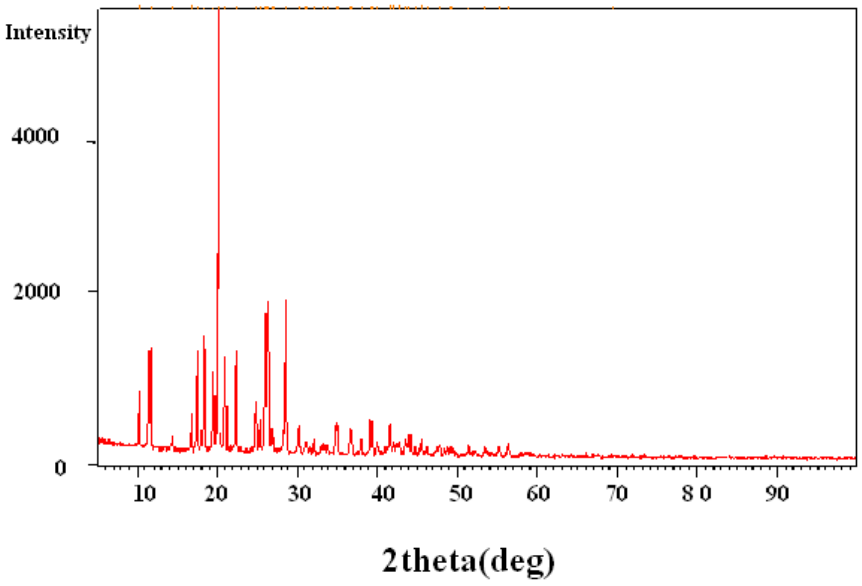

Fig. 3 X-Ray powder diffraction pattern of Crystalline Captopril

\subsection{Molecular Mobility-Relaxation Dynamics.}

Fig. 4 shows the dielectric loss spectra obtained during heating of amorphous Captopril from temperature123.15K to $373.15 \mathrm{~K}$ in different steps. To get the clear picture of the relaxation processes, the entire spectra is divided into two parts. Fig. 4a shows the relaxation above $T_{g}$, where structural $(\alpha)$ relaxation dominates and gives the changes in the structure of the investigated sample. The liquid-glass transition occurs at a relaxation time $\tau_{\alpha}=100$ sec. As temperature increases $\boldsymbol{\alpha}$ - processes peak moves towards higher frequencies indicating the increase of molecular mobility of the system (fig. 4a). At 343.15K the dielectric strength of $\alpha$ - process starts decreasing showing the indication of onset crystallization of the sample. It is concluded that structural relaxation is also responsible for devitrification of the drug other than secondary process [25] .Fig.4b shows the spectra collected below $\mathrm{T}_{\mathrm{g}}$ that is in the glassy state of Captopril. Only secondary relaxations are seen in this region, because $\alpha$-relaxation is too slow to be measured [18]. It is clear from fig. $4 \mathrm{~b}$, secondary relaxations moves towards lower frequencies with decreasing temperature. This finding proves that molecular mobility is present even in the glassy state of this drug.

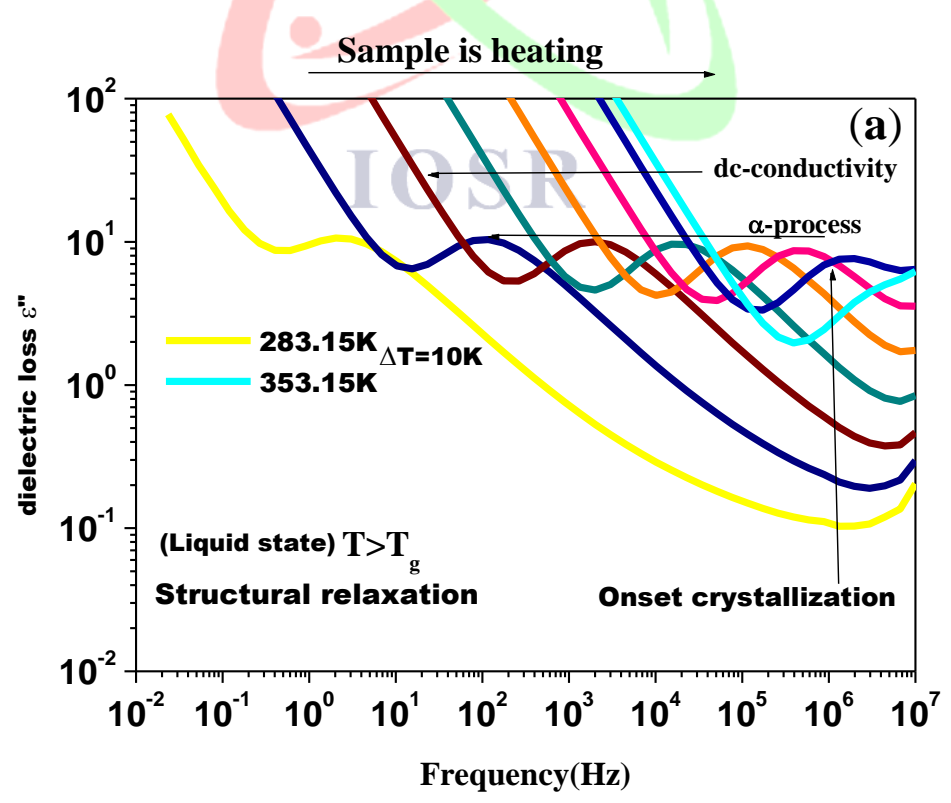


IOSR Journal of Pharmacy

Vol. 2, Issue 3, May-June, 2012, pp.479-484

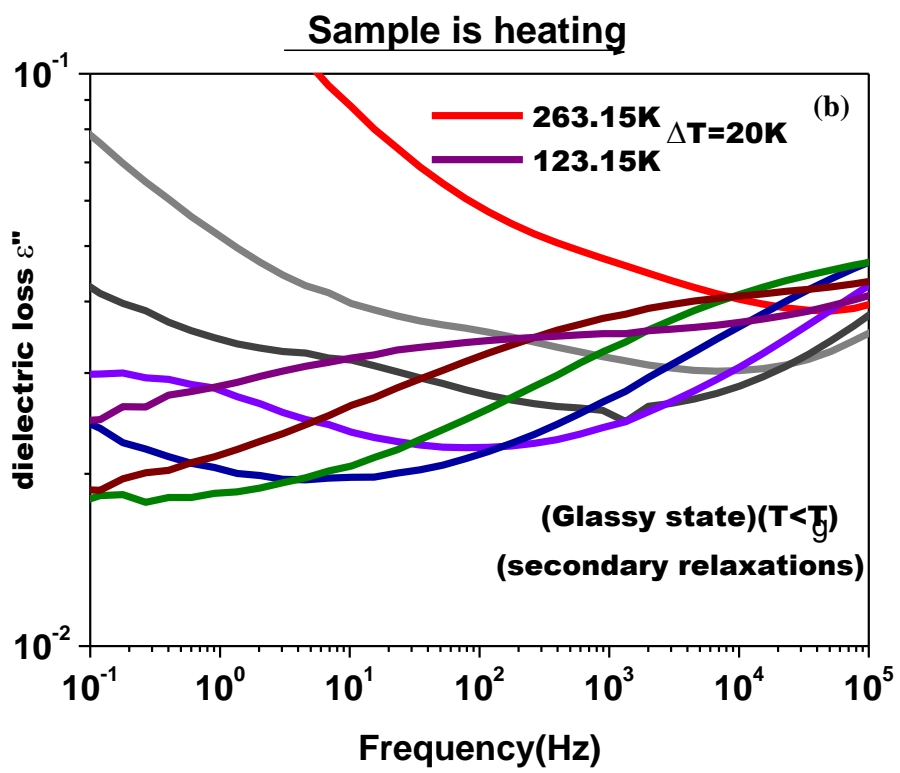

Fig. 4 Dielectric loss spectra of Captopril at different temperatures (a) above (b) below $\mathrm{T}_{\mathrm{g}}$

The temperature dependence of the $\alpha$-relaxation is often described by Vogel-Fulcher-Tamman-Hesse [VFTH] equation

$$
<\tau>=\tau_{V F} e^{B /\left|T-T_{0}\right|}
$$

with $\tau_{\mathrm{VF}}, \mathrm{B}$, and $\mathrm{T}_{0}$ as parameters. Using VFTH expression fragility $(\mathrm{m})$ can be calculated as

$$
m=\frac{d \log \tau}{d\left(T_{g} / T\right)} / T=T_{g}
$$

Fragility values normally lies between $m=16$ for strong systems and $m=200$ for fragile systems [4].

And for Captopil it is estimated as 84, and classify it as a fragile glass former. A general means of classifying glasses in terms of temperature dependence of molecular motions would be of great use to pharmaceutical materials scientists.

\section{CONCLUSIONS}

Calorimetric analysis proved the glass forming ability of Captopril. The existence of hydrogen bonding in the amorphous state is verified by IR spectroscopy and found to be stronger in the amorphous state than that of the crystalline state. Fragility is a key factor in determining the stability of a pharmaceutical drug and for Captopril it is found to be 84 and shows it is a fragile glass former. The dielectric study of Captopril in the amorphous state is done by BDS covering a broad frequency range. The spectra is found to be complex. Above the glass transition temperature along with $\alpha$-process dc-conductivity associated with translation motion of ions is also present. Also the examined drug starts crystallizing at $343.15 \mathrm{~K}$ showing the role of structural $(\alpha)$ relaxation in the devitrification process. Molecular mobility which is found to be present below glass transition temperature plays an important role in the devitrification of the drug. A better understanding of molecular dynamics in the glassy state of a pharmaceutical drug is very useful for the safety processing, and storage with predictable stability for a long period of time. Our observation might be useful for increasing the shelf life of this API in the amorphous form.

\section{ACKNOWLEDGMENTS}

We thank IIT Powai, Mumbai, CIL, NIPER Mohali Punjab, Chethana Pharmaceuticals, Kerala, for providing experimental facility. The authors gratefully acknowledge Mr.Mohith Agarwal of MEMS Dept. IIT Mumbai for BDS analysis, and Dr. Jisha of MES Keveeyam College for discussion of FTIR. S.U acknowledges UGC for selecting under FIP Scheme. 


\section{REFERENCES}

[1] Suga H, Seki S. Thermodynamic Investigation On Glassy State Of Pure Simple Compounds. J.Non. Cryst Solids. 16; (1974); 171-194.

[2] Wu L. Relaxation mechanisms in a benzyl chloride-toluene gas. Phys.Rev B. 43; (1991); 9906-9915.

[3] Johari JP, Goldstein M. Viscous Liquids and the Glass Transition.III. Secondary relaxation in aliphatic alcohols and other non rigid molecules. J.chem.Phys. 55; (1971); 4245-4252.

[4] Boehmer R, Ngai KL, Angel CA, Plazek DJ. Nonexponential relaxations in strong and Fragile glass formers. J. Chem. Phys. 99; (1993); 4201-4209.

[5] Hancock BC, Zografi, G. Characteristics and Significance of the Amorphous State in Pharmaceutical Systems. J.Pharm.Sci. 86; (1997); 1-12.

[6] Zhou D, Zhang GGZ, Law D, Grant DJW, Schmitt EA. Physical Stability of Amorphous Pharmaceuticals: Importance of Configurational Thermodynamic Quantities and Molecular Mobility. J.Pharm.Sci. 91; (2002); 1863-1872.

[7] Yoshioka S, Aso Y,Miyazai T. Negligible contribution of Molecular Mobility to the Degradation Rate of Insulin Lyophilized with Polyvinylpyrrolidone. J.Pharm.Sci. 95; (2005); 939-943.

[8] Hancock BC, Parks M. What is the True Solubility Advantage for Amorphous Pharmaceuticals?. Pharm.Res. 17; (2000); 397-404.

[9] Gupta P, Chawla G, Bansal AK. Physical Stability and Solubility Advantage from Amorphous Celecoxib: The Role of Thermodynamic Quantities and Molecular Mobility. Mole. Pharmaceutics. 1; (2004); 406-413.

[10] Correia T, Moura Ramos JJ, Descamps M, Collins G. Molecular Mobility and Fragility in Indomethacin: A Thermally Stimulated Depolarization Current Study. $\quad$ Pharm.Res. 18; (2001); 1767774.

[11] Kaminski K, Kaminska E, Adrjanowicz K, Grzybowiska K, Wlodarczyk P, Paluch M, Burian A, Ziolo J, lepek P, Mazgalski J, Sawicki W. Dielectric Relaxation Study on Tramadol Monohydrate and Its Hydrochloride Salt. J.Pharm.sci. 99; (2009); 94-106.

[12] Alie J Menegotto J, Cardon P, Dupla H, Caron A, Lacabanne C, Bauer M. Dielectric Study of the Molecular Mobility and the Isothermal Crystallization Kinetics of an Amorphous Pharmaceutical Drug Substance. J.Pharm.sci. 93; (2003); 218-233.

[13] Shahin Thayyil M, Capaccioli S, Daniele Prevosto, Ngai K L. Is the Johari-Goldstein $\beta$-relaxation universal?. Philosophical Magazine. (2008); ISSN: 1478-6443.

[14] Capaccioli S, Shahin Thayyil M, Ngai K L. Critical issues of current research on the Dynamics leading to Glass Transition. J. Phys. Chem. B. (2008). 112, 16035-49 ISSN: 1478-6443.

[15] Adrjanowicz K, Wojnarowska Z, Wlodarczyk P, Kaminski K, Paluch M, Mazgalski J. Molecular mobility in liquid and glassy states of Telmisartan (TEL) studied by Broadband Dielectric Spectroscopy. Eur.J.Pharm.Sci. 38; (2009); 395-404.

[16] Bra's AR, Noronha JP, Antunesn A.M.M, Cardoso MM, Scho“nhals A, Affouard F, Dioni'sio M, Correia NT. Molecular Motions in Amorphous Ibuprofen As studied by Broadband Dielectric Spectroscopy. J.Phys. Chem. B. 112; (2008); 11087-11099.

[17] Tripathi KD (MD) Essentials of Medical Pharmacology (jape brothers Medical publications (P) Ltd, (2008) page no 483.

[18] Grzybowska K, Paluch M, Grzybowski A, Wojnarowska Z, Hawelek L, Kolodziejczyk K. Molecular Dynamics and Physical Stability of Amorphous Anti-Inflammatory Drug: Celecoxib. J. Phys. Chem. B. 114; (2010); 12792-12801.

[19] Tang XC, Pikal MJ, Taylor LS. A Spectroscopic Investigation of Hydrogen Bond Patterns in Crystalline and Amorphous Phases in Dihydropyridine Calcium Channel Blockers. Pharm. Res. 19; (2002); 477- 483.

[20] Taylor LS, Zografi G .Spectroscopic Characterization of Interaction between PVP and Indomethacin in Amorphous Molecular Dispersions. Pharm.Res. 14; (1997); 1691-1698.

[21] Marsac PJ, Konno H, Taylor LS. A Comparison of the Physical Stability of Amorphous Felodipine and Nifedipine Systems. Pharm. Res. 23; (2006); 2306-2316.

[22] Indian Pharmacopoeia 2007 page no 849.

[23] Pajula K, Taskinen M, Lehto VP, Ketolainen J, Korhonen O. Predicting the Formation and Stability of Amorphous Small Molecule Binary Mixtures from 99 Computationally Determined Flory-Huggins Interaction Parameter and Phase Diagram. Mol. Pharmaceutics. 7; (2010); 795-804.

[24] Silverstein, R.M.; Bassler, G.C.; Morrill, T.C.; Spectrometric Identification of Organic Compounds. (Fifth edition. John Wiley\&Sons: Singapore1991).

[25] Grzybowska K, Paluch M, Wlodarczyk P, Grzybowski A, Kaminski K, Hawelek L. Enhancement of Amorphous Celecoxib Stability by Mixing It with Octaacetylmaltose: The Molecular Dynamics Study. Mol.Pharm. (2012), A-K. 